\title{
Attosecond quantum entanglement in neutron Compton scattering from water in the $\mathrm{keV}$ range
}

\author{
C. A. Chatzidimitriou-Dreismann ${ }^{1}$ \\ ${ }^{1}$ Institute of Chemistry, Faculty II, Technical University of Berlin, D-10623 Berlin, \\ Germany. Email: dreismann@chem.tu-berlin.de
}

\begin{abstract}
Scattering of neutrons in the 24-150 keV incident energy range from $\mathrm{H}_{2} \mathrm{O}$ relative to that of $\mathrm{D}_{2} \mathrm{O}$ and $\mathrm{H}_{2} \mathrm{O}-\mathrm{D}_{2} \mathrm{O}$ mixtures was reported very recently. Studying time-of-flight integrated intensities, the applied experimental procedure appears to be transparent and may open up a novel class of neutron experiments regarding the "anomalous" scattering from protons, firstly observed in our experiment at ISIS in the 5-100 eV range. The keV-results were analyzed within standard theory, also including (1) multiple scattering and (2) the strong incident-energy dependence of the neutron-proton cross section $\sigma_{H}\left(E_{0}\right)$ in this energy range. The analysis reveals a striking anomalous ratio of scattering intensity of $\mathrm{H}_{2} \mathrm{O}$ relative to that of $\mathrm{D}_{2} \mathrm{O}$ of about $20 \%$, thus being in surprisingly good agreement with the earlier results of the original experiment at ISIS.
\end{abstract}

Keywords: neutron Compton scattering, attosecond physics, quantum entanglement

\section{Introduction}

Several neutron Compton scattering (NCS) experiments on liquid and solid samples containing protons or deuterons show a striking anomaly, i.e. a shortfall in the intensity of energetic neutrons scattered by the protons; cf. [1, 2, 3, 4]. E.g., neutrons colliding with water for just $100-500$ attoseconds $\left(1\right.$ as $\left.=10^{-18} \mathrm{~s}\right)$ will see a ratio of hydrogen to oxygen of roughly 1.5 to 1 , instead of 2 to 1 corresponding to the chemical formula $\mathrm{H}_{2} \mathrm{O}$. Due to the large energy and momentum transfers applied, the duration of a neutron-proton scattering event (the so-called scattering time) is a fraction of a femtosecond which is extremely short compared to the usual condensed-matter relaxation times.

Recently this new effect has been independently confirmed by electron-proton Compton scattering (ECS) from a solid polymer [3 4, 5]. The similarity of ECS and NCS results is striking because the two projectiles interact with protons via fundamentally different forces, i.e. the electromagnetic and strong forces.

Due to its novelty and far-reaching consequences, however, this effect has been the focus of various criticisms, cf. [6, 7]. Therefore, considerable work to identify possible sources of experimental and data analysis errors was made during the 
last five years, which succeeded to demonstrate the excellent working conditions of the spectrometer Vesuvio; see [8] for an account in detail. Extending these investigations, the complete "exact formalism" of data analysis [6] was applied to NCS-data by Senesi et al. [9], for the first time. Analysis of time-of-flight (TOF) spectra from solid $\mathrm{HCl}$ revealed the existence of an "anomalous" decrease of the scattering intensity from $\mathrm{H}$ by $34 \%$. Moreover, this result was found to be in excellent agreement with the corresponding outcome of the standard data analysis procedure applied at ISIS [9]. Additionally, the mentioned standard NCSdata analysis method [8] was successfully compared with a newly proposed (by B. Dorner, ILL) model-free data-analysis procedure, the latter being independent of the form of the momentum distribution and the resolution function [10].

\section{NCS in the keV range}

In a novel experiment by Moreh et al. [11], scattering results of neutrons from $\mathrm{H}_{2} \mathrm{O}$ relative to that of $\mathrm{D}_{2} \mathrm{O}$ and $\mathrm{H}_{2} \mathrm{O}-\mathrm{D}_{2} \mathrm{O}$ mixtures were reported, in the incident energy range about $24-150 \mathrm{keV}$. This experiment was carried out to search for the aforementioned anomalous decrease [1] in the neutron scattering intensity from protons (relative to that from deuterons) at interaction times in the attosecond regime. It is important to note that the energy range here is about 3000 times larger than that of our original neutron Compton scattering (NCS) experiment carried out with the electron volt spectrometer eVS (newly: Vesuvio) at the neutron spallation source ISIS [1].

In clear contrast to the original NCS results [1], Moreh et al. claimed that the results in $\mathrm{keV}$ range exhibited no anomalous behavior. It was concluded that within an overall statistical accuracy of $3 \%$ there is no evidence for any deviation from the ratios of scattering intensities conventionally calculated on the basis of the tabulated total neutron cross sections [11]. In addition, it was correctly argued that one would have to shake some well established notions in physics to explain the aforementioned scattering anomaly.

Our theoretical analysis of these experimental data, and in particular their comparison with predictions of standard NCS theory [12], is considered in the following. This analysis reveals a striking anomalous ratio of scattering intensity of $\mathrm{H}_{2} \mathrm{O}$ relative to that of $\mathrm{D}_{2} \mathrm{O}$ of about $20 \%$. Extending the first analysis of single scattering events [13], it will be shown that $(a)$ neither multiple scattering $(b)$ nor the strong dependence of the proton total cross section $\sigma_{H}\left(E_{0}\right)$ on incident energy $E_{0}$, in the considered $\mathrm{keV}$ range, do considerably affect the anomaly under investigation.

\subsection{Experimental}

First of all, it is important to notice that both setups, i.e. that of the new keVneutron experiment at the Rensselaer Polytechnic Institute (RPI) [11] and that of Vesuvio at ISIS [8], are basically similar and thus the interpretation of their results ought to be based on the same basic theory. The following related remarks should be helpful:

(i) Both are so-called inverse geometry time-of-flight (TOF) setups; i.e., the final energy $E_{f}$ of the measured neutrons is fixed, the neutron initial energy $E_{0}$ is "continuous", and the scattered neutrons are analyzed using a "filter" (of Fe at 
RPI, with $E_{f}=24.3( \pm 1.1) \mathrm{keV}$ ) or "analyzer foil" (of Au at ISIS, with $E_{f}=$ $4.91( \pm 0.14) \mathrm{eV})$.

(ii) The range of scattering angles $\theta$ is similar in both setups, (i.e. one detector integrating over $\theta=25^{\circ}-65^{\circ}$ at RPI; 32 detectors measuring at various scattering angles in the range $35^{\circ}-67^{\circ}$ at ISIS).

( iii) As a consequence, and according to standard theory [12], the characteristic neutron-proton scattering time in the $\mathrm{keV}$ range is shorter by a factor of about 70 [11] as compared to that of the Vesuvio-setup. In view of some of the mentioned theoretical models (see below), however, this difference of scattering times may not preclude the appearance of the considered effect in the keV range.

(iv) The Impulse Approximation (IA) can be safely assumed to be exact in the $\mathrm{keV}$ range [11], and it is already known to be sufficiently fulfilled in the $\mathrm{eV}$ range of Vesuvio [8, 12]. In simple terms, each neutron scatters from a single nucleus (of $\mathrm{H}, \mathrm{D}$ or $\mathrm{O}$ ).

$(v)$ The setup at RPI cannot resolve the neutrons scattered from different nuclei and yields instead an integrated scattering signal arising from the $(\mathrm{O}, \mathrm{H}$ and/or D) nuclei of the liquid samples. The ISIS setup, however, provides a H-recoil peak well resolved from that of $\mathrm{D}$ or $\mathrm{O}$, but this does not represent any significant difference that would prevent comparison of results. (E.g., one can simply integrate over the individual peak intensities, when required).

(vi) Furthermore, note that the scattering intensity, measured by a single detector, is represented by the area of the $24.3 \mathrm{keV}$ line in the TOF spectra, i.e. by adding up the total number of counts in the $24.3 \mathrm{keV}$ peak [11]. An independent fission detector, placed inside a separate beam tube, was employed as a neutron flux monitor and served to normalize the TOF spectra. After subtracting the background from each signal, the intensity ratios are taken, as presented in Fig. 3 of [11]. The measurements were repeated about five times, always giving about the same results within statistics (personal communication).

(vii) Already here it should be emphasized that, as already stressed in Ref. [11], multiple scattering effects do not affect the ratio of scattering intensities from the two samples; see also below.

\subsection{Single scattering events}

Recognizing the crucial importance of these novel keV-experimental results, we analyzed in detail [13] the data processing scheme indicated in Ref. [11] on the basis of standard scattering theory at large energy transfers [8, 12], where the IA is valid; cf. point $(i v)$ above. Some omissions in [11] have been revealed and then amended, thus leading to a radical revision of the main finding and conclusion of Ref. [11]. In accordance with the preceding point (vii), in that calculation [13] we considered single scattering events only. That calculation [13], which also took into account the strong dependence of the proton total cross section $\sigma_{H}\left(E_{0}\right)$ on incident energy $E_{0}$ [14], contains no fitting parameter.

A full account in detail of these investigations is presented in Ref. [13]. The results are summarized in Fig. 1 and lead to a radical revision of the main finding and conclusion of Ref. [11]: The correct $\mathrm{keV}$-data reduction reveals a strongly anomalous ratio of scattering intensity of $\mathrm{H}_{2} \mathrm{O}$ with that of $\mathrm{D}_{2} \mathrm{O}$ of about $20 \%$, thus being in surprisingly good agreement with the associated results of the original ISIS-experiment [1]. 
Our results [13] were confirmed independently by Monte Carlo calculations of Mayers [15], applying the routines for NCS-data analysis available at ISIS. Also these calculations contain no fitting parameters.

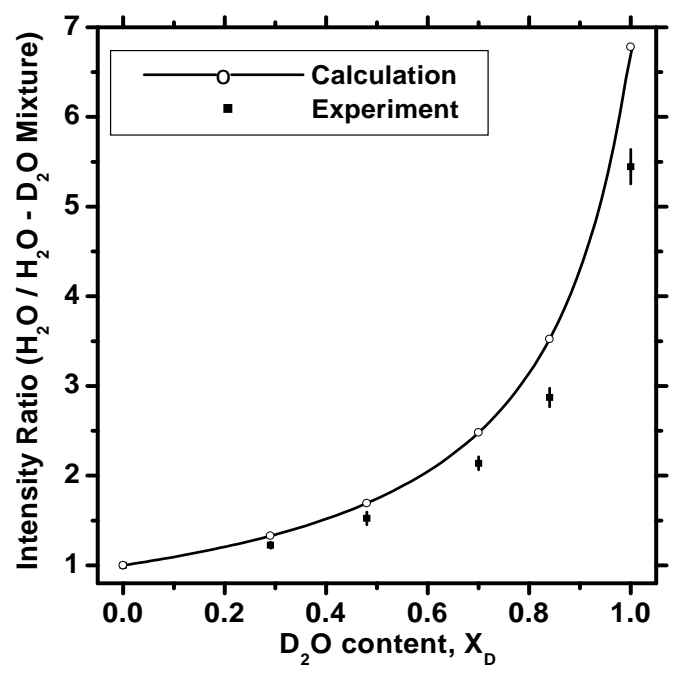

Figure 1: Intensities of single scattering events [13]. Measured (full squares with error bars) - taken from Ref. [11] - and corrected calculation (solid line, open circles) for $\theta=45^{\circ}$ of scattered intensity ratios versus $X_{D}$, the $\mathrm{D}_{2} \mathrm{O}$ concentration in the $\mathrm{H}_{2} \mathrm{O}-\mathrm{D}_{2} \mathrm{O}$ mixture. The discrepancy between experimental data and prediction of conventional theory is clearly discernible for data with $X_{D} \geq 0.5$. The calculated intensity ratio for pure $\mathrm{H}_{2} \mathrm{O}$ and pure $\mathrm{D}_{2} \mathrm{O}$ is ca. $23 \%$ higher than the experimental one.

\subsection{Double scattering events - Energy dependence of $\sigma_{H}$}

The thickness of all samples in the $\mathrm{keV}$ experiment was $d=0.18 \mathrm{~cm}$. However, as already mentioned under point ( $v i i$ ) above, is was shown that multiple scattering effects did not affect the considered ratio of scattering intensities; explicitly: "The effect on the ratio of scattering intensities from the two samples is $<1 \%$ and was neglected."; see page 4 of Ref. [11].

Applying the Monte Carlo routines available at ISIS, J. Mayers analyzed the multiple scattering effects on water and $\mathrm{D}_{2} \mathrm{O}$, for the sample thickness of $d=0.18$ $\mathrm{cm}$ [15]. In these calculations, however, the proton total cross section $\sigma_{H}$ was kept constant (e.g. 15.7 barn). The obtained results clearly showed that, in the $\mathrm{H}_{2} \mathrm{O}$ case, the single scattering events are attenuated due to the considerable sample thickness. (Note that the transmittance of the $\mathrm{H}_{2} \mathrm{O}$ sample (for $E_{0}=48.6 \mathrm{keV}$ ) is ca. $81 \%$, see numerical calculations below). But, the crucial point here was that the twice scattered neutrons were found to go mainly at forward scattering angles, and that $\theta \approx 45^{\circ}$ is at the center of the the double scattering events in the forward angle range. Thus, as sample attenuation increases, multiple scattering also increases. As a result, and including multiply scattered neutrons, the ratio of 
scattering intensity of $\mathrm{H}_{2} \mathrm{O}$ relative to that of $\mathrm{D}_{2} \mathrm{O}$ was calculated to be about 6.4 [15], i.e. thus about $17 \%$ larger than the associated experimental value of 5.5 [11].

Nevertheless, and due to the potentially far-reaching consequences of the provided new keV experimental results, we extended the aforementioned analysis [13] and Monte Carlo simulations [15] by including the strong dependence of the proton total cross section $\sigma_{H}$ on incident energy $E_{0}[14]$ into the calculations of double scattering.

This dependence could be important, for the following reason. Neutrons which are not scattered once into the detector are multiply scattered. Such neutrons may have very high incident energies $E_{0}$, before they end up with final energy $E_{f}=$ $24.3 \mathrm{keV}$ and become measured. Therefore they would have a much lower crosssection and hence the probability of scattering for these neutrons may be very low. Consequently it is conceivable that the multiple scattering contributions from protons could be significantly lower than those conventionally calculated on the basis of a fixed proton-neutron cross section (say, e.g., with $\sigma_{H}=15.7$ barn, being valid for $E_{0}=48.6 \mathrm{keV}$ ).

Here it should be emphasized that the existing Monte Carlo routines for the calculation of multiple scattering effects cannot handle the present case of $\sigma_{H}$ depending on incident energy $E_{0}$; cf. [16]. The reason for this should be that, up to ca. $1 \mathrm{keV}$, the "free" total cross section of $\mathrm{H}$ remains constant, and the thus far existing NCS investigations were well within this energy range.

To simplify the derivations and pinpoint their main physical aspects, we consider here double scattering from the water's protons only. Let $N_{H}$ be the number of $\mathrm{H}$ atoms per unit volume and

$$
\mu\left(E_{x}\right) \equiv N_{H} \sigma_{H}\left(E_{x}\right)
$$

at "incident" neutron energy $E_{x}$ before the $x$-th neutron-proton collision. Let $\beta$ be the scattering angle of the impinging neutron with initial energy $E_{0}$ due to the first neutron-proton collision, and $\phi$ being the associated azimuthal angle. (As usual, polar coordinates are used here.) Furthermore, let $\chi$ be the angle between the two neutron-velocity vectors before and after the second scattering event. We consider neutrons with a total scattering angle $\theta$ and with final energy $E_{f}=24.3 \mathrm{keV}$ going into the detector. As is well known [17, 18], the "intermediate" scattering angle $\chi$ depends on both $\beta$ and $\theta$. A short calculation yields

$$
\cos \chi=\cos \theta \cos \beta+\sin \theta \sin \beta \cos \phi
$$

As conventionally, we consider here an infinite slab geometry for the sample, with thickness $d$, and the incident neutron beam in the direction $z$ being perpendicular to the slab.

According to standard theory [8, 19], the neutron energy $E_{1}$ between first and second neutron-proton collision is

$$
E_{1}=\frac{E_{f}}{\cos ^{2} \chi}
$$

and the associated initial energy is

$$
E_{0}=\frac{E_{f}}{\cos ^{2} \chi \cos ^{2} \beta}
$$


Recall that here $E_{f}=24.3 \mathrm{keV}$. Thus, for some double-scattering events, $E_{0}$ may be very large and the cross section $\sigma_{H}\left(E_{0}\right)$ for the first scattering process very small [14].

Furthermore we need the three attenuation factors (also called self shielding or absorption corrections) [20] for the neutron propagation through the sample:

$$
U_{0}=\exp \left[-\mu\left(E_{0}\right) z_{0}\right]
$$

$z_{0}$ being the neutron path in the sample until its first collision,

$$
U_{1}=\exp \left[-\mu\left(E_{1}\right) s_{1}\right]
$$

$s_{1}$ being the neutron path in the sample between its first and second collision, and finally

$$
U_{f}=\exp \left[-\mu\left(E_{f}\right) \frac{d-z_{0}-s_{1} \cos \beta}{\cos \theta}\right]
$$

for the path between the second collision and the exit from the sample, in the fixed scattering direction $\theta$ towards the detector.

Furthermore, the probability for neutron scattering under scattering angle $\psi$ in the neutron-proton collision is proportional to $\cos \psi$; see Eq. (1.88) of [19].

The intensity of impinging neutrons in the $\mathrm{keV}$ experiment is proportional to $I\left(E_{0}\right)=E_{0}^{-0.65}[11]$.

As a result, the above partial processes lead straightforward to the following expression for the probability $P_{d s e}$ of a double-scattered neutron with final energy $E_{f}=24.3 \mathrm{keV}$ measured with the detector at scattering angle $\theta$ :

$$
\begin{aligned}
P_{d s e} \propto \int I & \left(E_{0}\right)\left[U_{0} \mu\left(E_{0}\right) \sin \beta \cos \beta\right] \\
& \times\left[U_{1} \mu\left(E_{1}\right) \cos \chi\right] U_{f} d z_{0} d \beta d \phi d s_{1}
\end{aligned}
$$

(The squared brackets are for convenience only; they include the factors belonging to each scattering event.) The factor $\sin \beta$ is due to the scattering after the first collision [19]. Note the presence of the factors $\mu\left(E_{0}\right)$ and $\mu\left(E_{1}\right)$, which are associated with the scattering probability at the two scattering points.

\subsection{Main result - strongly anomalous $R$}

Note that all these equations are valid for the case of energy-dependent $\sigma_{H}\left(E_{0}\right)$. The probabilities for the twice scattered neutrons can be calculated with Eq. (8). The evaluation can be carried out with the Monte Carlo integration method; see e.g. Sect. 7.6 of [21].

The following observation is now of crucial importance. The same equation (8) can also be evaluated for the case of fixed cross section $\sigma_{H}=$ const. (For this, one simply has to fix the value of $\sigma_{H}$ in Eq. 1.) Thus the results of both calculations can be directly compared, as they are shown in Fig. 2. This comparison shows quantitatively the effect of the energy dependence of $\sigma_{H}$, for various scattering angles. The results obtained with fixed $\sigma_{H}$-value confirm the aforementioned Monte Carlo calculations of Mayers. The effect of the energy dependence $\sigma_{H}\left(E_{0}\right)$ leads, at $\theta=45^{\circ}$, to a slight decrease of the twice scattered neutrons of about $23 \%$ only. 


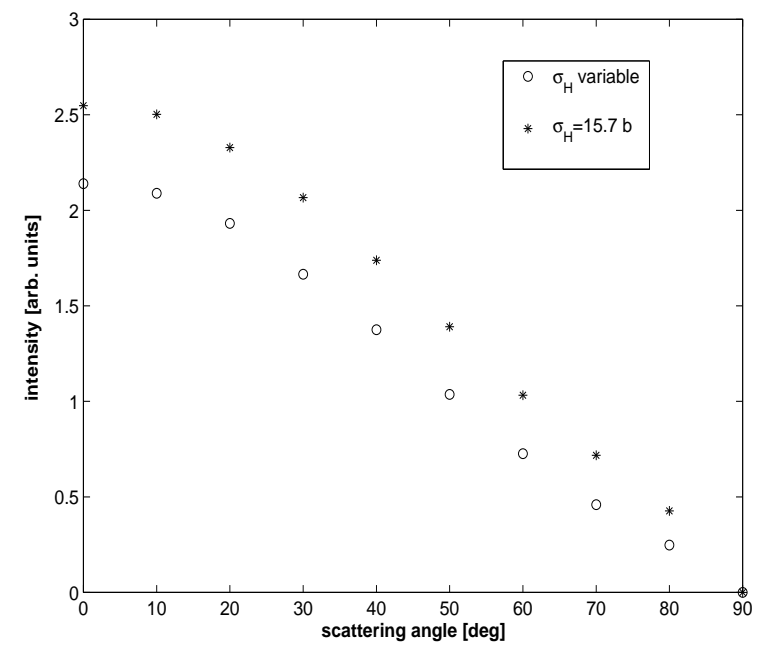

Figure 2: Intensity of double scattered neutrons from protons of pure $\mathrm{H}_{2} \mathrm{O}$ as a function of total scattering angle $\theta$, calculated with Eq. (8). Asterisks: Calculation with fixed cross section $\sigma_{H}=15.7$ b. Circles: Energy-dependent $\sigma_{H}\left(E_{0}\right)$, with $E_{0}$ determined by Eq. (4). Note the "forward" orientation of the twice scattered neutrons, which is equal in both calculations. The effect of the energy dependence of $\sigma_{H}\left(E_{0}\right)$ leads to a decrease of the twice scattered neutrons of about $23 \%$ at $\theta=45^{\circ}$. This amounts to ca. $3.5 \%$ of the complete intensity only; see the text.

Furthermore, calculation of the single-scattered neutron intensity at $\theta$ was carried out by

$$
P_{s s e} \propto \int_{0}^{d} I\left(E_{0}\right)\left[U_{0}^{\prime} \mu\left(E_{0}^{\prime}\right) \cos \theta\right] U_{f}^{\prime} d z_{0}
$$

with $E_{0}^{\prime}=E_{f} / \cos ^{2} \theta, U_{0}^{\prime}=\exp \left[-\mu\left(E_{0}^{\prime}\right) z_{0}\right]$ and

$$
U_{f}^{\prime}=\exp \left[-\mu\left(E_{f}\right) \frac{d-z_{0}}{\cos \theta}\right]
$$

(This integral can be calculated analytically, too [20].) Comparison of the above results showed that the ratio of double- to single-scattered neutrons is about 0.15 at $\theta=45^{\circ}$. Thus we may proceed to the following crucial conclusion: If one approximately neglects higher-order scattering events (which were estimated to be about $2-3 \%$ of the total scattering), then the aforementioned $23 \%$-reduction of the double-scattered neutrons amounts to ca. $3.5 \%$ of the complete intensity at $\theta=45^{\circ}$. This furthermore implies that - neglecting double-scattering from $\mathrm{D}_{2} \mathrm{O}$ the earlier calculated ratio $R=6.78$ [13] should be slightly reduced to $R=6.55$, which is still about $19 \%$, and thus significantly, larger than the experimentally measured ratio $R_{\exp }=5.5$

Moreover, if one would also take into account double scattering events from $\mathrm{D}_{2} \mathrm{O}$, it is obvious that the considered ratio $R$ of integrated scattering intensities from $\mathrm{H}_{2} \mathrm{O}$ and $\mathrm{D}_{2} \mathrm{O}$ will be slightly increased again. Thus we may safely conclude 
that the theoretically predicted value of the ratio $R$ still remains about $20 \%$ larger than the experimental one $R_{\text {exp }}=5.5$.

This crucial result is fully in line with the previous calculations based on single scattering events only [13]. It shows that inclusion of double scattering, as well as the strong dependence of the proton total cross section $\sigma_{H}$ on incident energy $E_{0}$ into the calculations, confirm again the anomaly of the intensity ratio $R$. As the overall errors of the keV-measurements were about $3 \%$ while the actual statistical errors were about $2 \%$ [11], one concludes that the revealed anomaly of ca. $20 \%$ is highly significant.

\section{Theoretical remarks}

Originally it has been proposed [1] that the considered effect is caused by shortlived and spatially restricted entanglement. Since the typical neutron- and electronproton interaction time in NCS and ECS (i.e. $\tau_{s c}$ ) lies within the attosecond range, it is expected that decoherence may still not be fully effective. Published theoretical models attribute this effect to:

$(A)$ Modification of scattering due to "identity of particles" in the scattering system. The influence on scattering of entanglement of the spin and spatial degrees of freedom of identical particles (i.e., quantum exchange correlations) has been stressed in Refs. [22]. In particular, NCS from pairs of protons and deuterons has been calculated.

$(B)$ Contribution of electronic degrees of freedom to the dynamics of a struck proton (deuteron) interacting with its environment. E.g., breakdown of the BornOppenheimer (BO) approximation in the final state of the NCS process and (B.1) additional excitations of the electronic system [23, 24], and/or (B.2) decoherence accompanying short-lived spatial entanglement of a struck proton with adjacent electrons and perhaps also nuclei [25]. In the models of this category, spin entanglement and/or quantum exchange correlations between identical particles play no role.

To test certain contradictory predictions of these theories, we recently measured by NCS $(a)$ the equimolar $\mathrm{H}_{2}-\mathrm{D}_{2}$ mixture and $(b)$ the mixed-isotope system HD (liquids, both at $20 \mathrm{~K}$ ). The crucial result was that both systems reveal the same, strong anomalous shortfall (about 30\%) of the ratio $\sigma_{H} / \sigma_{D}$ of $\mathrm{H}$ and D cross-sections [26]. Since HD exhibits no exchange correlations, this result demonstrates for the first time that these correlations play no significant role in this effect, thus refuting corresponding theoretical models claiming its interpretation. This conclusion is in line with a recently presented full calculation of the scattering function $S(q, \omega)$ by Sugimoto et al. [27], who found that indistinguishability of particles cannot represent the physical origin of the observed effect.

In contrast, our findings [26] are consistent with alternative theoretical models of category $(B)$, in which attosecond dynamics of electronic degrees of freedom - e.g. via violation of the BO approximation - is considered to participate significantly to the dynamics of an elementary neutron-proton (-deuteron) scattering process. The scattering times of NCS and ECS are similar to the characteristic time of "electron motion", so that the Born-Oppenheimer approximation is not applicable here; cf. Ref. [2a]. However, it should be stressed that a detailed treatment of the BO failure and a quantitative estimate of its possible contribution to the considered NCS-anomaly does not exist yet, as convincingly discussed in Ref. [28]. 


\section{Additional remarks}

The preceding derivations concerning the new keV experimental results [11] have various far-reaching theoretical and experimental consequences. Some of them are as follows.

First, it should be stressed that the intensity ratios determined in this experiment can only provide information about a possible difference between the scattering behavior of $\mathrm{H}$ and $\mathrm{D}$, and not about that of $\mathrm{H}$ alone. In view of several theoretical works [23, 24, 25], it may be expected that, due to the high energy transfers, both protons and deuterons should violate the BO approximation, thus leading to the above "anomaly" for both $\mathrm{H}$ and $\mathrm{D}$. Thus, the measurement of the scattering intensity ratio $R_{\text {exp }}$ of $\mathrm{H}_{2} \mathrm{O}$ relative to that of $\mathrm{D}_{2} \mathrm{O}$ is not appropriate for the search of the anomalous scattering effect under consideration. Obviously, an amended $\mathrm{keV}$ experiment would be to measure the scattering intensity ratio of $\mathrm{H}_{2} \mathrm{O}\left(\mathrm{D}_{2} \mathrm{O}\right.$, and their mixtures) relative to that of a heavy metal sample, e.g. $\mathrm{Pb}$. This experiment was recently proposed by the author.

Second, already earlier NCS from D-containing materials have shown that also D exhibits a small anomalous shortfall of scattering intensity. E.g. in [2c] was reported that this shortfall in $\mathrm{NbD}_{0.8}$ was about $10 \%$. Furthermore, several NCS measurements on pure $\mathrm{D}_{2} \mathrm{O}$ showed up a shortfall of the cross sections ratio $\sigma_{D} / \sigma_{O}$ of about $10-15 \%$ (unpublished data). Thus, the aforementioned $20 \%$ anomaly of the measured ratio $R_{\exp }$ in the $\mathrm{keV}$ experiment indicates that the integrated scattering intensity of pure $\mathrm{H}_{2} \mathrm{O}$ may exhibit an anomalous shortfall larger than $20 \%$. In view of these considerations, the aforementioned proposed experiment may be expected to yield an anomalous intensity ratio of $\mathrm{H}_{2} \mathrm{O}$ to $\mathrm{Pb}$ of the order of $25 \%$ and more.

Summarizing, we conclude that the considered scattering effect is present at both 5-100 eV [1] and 24-150 keV [11] ranges of incident energies. Obviously, the novel experiment [11] and its correct analysis established above open up new perspectives for neutron research on the above attosecond effect, and thus they may have far reaching consequences for current and future experimental and theoretical investigations.

\section{Acknowledgment}

I acknowledge partial support by the EU (the QUACS RTN) and by a grant from the Royal Swedish Academy of Sciences.

\section{References}

[1] C. A. Chatzidimitriou-Dreismann, T. Abdul-Redah, R. M. F. Streffer, and J. Mayers, Phys. Rev. Lett. 79 (1997) 2839.

[2] (a) C. A. Chatzidimitriou-Dreismann et al., J. Am. Chem. Soc. 123 (2001) 11945; (b) C. A. Chatzidimitriou-Dreismann et al., J. Chem. Phys. 116 (2002) 1511; (c) E. B. Karlsson et al., Europhys. Lett. 46 (1999) 617; (d) T. Abdul-Redah et al., Physica B 276-278 (2000) 824; (e) E. B. Karlsson et al., Phys. Rev. B 67, (2003) 184108. 
[3] C. A. Chatzidimitriou-Dreismann, M. Vos, C. Kleiner, and T. Abdul-Redah, Phys. Rev. Lett. 91 (2003) 057403.

[4] See e.g.: Physics Today, Sept. 2003, 9; Scientific American, Oct. 2003, 20.

[5] M. Vos, C. A. Chatzidimitriou-Dreismann, T. Abdul-Redah, and J. Mayers, Nucl. Instr. Meth. B 227 (2005) 233.

[6] (a) J. J. Blostein et al., Physica B 304 (2001) 357 (2001), and 334 (2003) 257; (b) J. J. Blostein et al., Phys. Rev. Lett. 90, (2004) 105302.

[7] R. A. Cowley, J. Phys. Condens. Matter 15 (2003) 4143.

[8] J. Mayers and T. Abdul-Redah, J. Phys. Condens. Matter 16, 4811 (2004).

[9] R. Senesi, D. Colognesi, A. Pietropaolo, and T. Abdul-Redah, Phys. Rev. B 72 (2005) 054119.

[10] M. Krzystyniak and C. A. Chatzidimitriou-Dreismann, Phys. Rev. B 72 (2005) 174117.

[11] R. Moreh, R. C. Block, Y. Danon, and M. Neumann, Phys. Rev. Lett. 94 (2005) 185301.

[12] G. I. Watson, J. Phys. Condens. Matter 8 (1996) 5955.

[13] C. A. Chatzidimitriou-Dreismann and M. Krzystyniak, Comment, submitted (2005).

[14] P. F. Rose and C. L. Dunford, "ENDF-102 Data Format and Procedures for the Evaluated Nuclear Data File ENDF-6", BNL-NCS-44945, Rev. 2, Brookhaven National Laboratory, 1997; see: www.nndc.bnl.gov.

[15] J. Mayers, personal communication (July 2005).

[16] J. Mayers, A. L. Fielding, and R. Senesi, Nucl. Instr. Meth. A 481 (2002) 454.

[17] G. H. Vineyard, Phys. Rev. 96 (1954) 93.

[18] A. K. Agraval, Phys. Rev. A 4 (1971) 1560.

[19] S. W. Lovesey, Theory of Neutron Scattering from Condensed Matter (Clarendon, Oxford, 1984).

[20] C. Windsor, Pulsed Neutron Scattering (Taylor \& Francis, London, 1981).

[21] W. H. Press, B. P. Flannery, S. A. Teukolsky, and W. T. Vetterling, Numerical Recipies - The Art of Scientific Computing (Cambridge Univ. Press, Cambridge, 1986).

[22] (a) E. B. Karlsson and S. W. Lovesey, Phys. Rev. A 61 (2000) 062714, and Phys. Scr. 65 (2002) 112; (b) E. B. Karlsson, Phys. Rev. Lett. 90 (2003) 095301.

[23] N. I. Gidopoulos, Phys. Rev. B 71 (2005) 054106.

[24] G. F. Reiter and P. M. Platzman, Phys. Rev. B 71 (2005) 054107.

[25] (a) C. A. Chatzidimitriou-Dreismann, Laser Physics 15 (2005) 780; (b) C. A. Chatzidimitriou-Dreismann and S. Stenholm, in: Decoherence, Entanglement and Information Protection in Complex Quantum Systems, V. M. Akulin, A. Sarfati, G. Kurizki and S. Pellegrin (eds.), pp. 555-562 (Springer, Dordrecht, 2005). 
[26] C. A. Chatzidimitriou-Dreismann, T. Abdul-Redah, and M. Krzystyniak, Phys. Rev. B 72 (2005) 054123.

[27] H. Sugimoto, H. Yuuki, and A. Okumura, Phys. Rev. Lett. 94 (2005) 165506.

[28] D. Colognesi, Physica B 358 (2005) 114. 